\title{
Une version effective du théorème de Briançon-Skoda dans le cas algébrique discret
}

\author{
par \\ M. Elkadi (Talence)
}

1. Introduction. Soient $P_{1}, \ldots, P_{m}, Q m+1$ polynômes en $n$ variables à coefficients complexes, avec $Q$ appartenant à la clôture intégrale locale de l'idéal engendré par $P_{1}, \ldots, P_{m}$; ceci signifie que pour tout $z_{0} \in \mathbb{C}^{n}$, il existe un voisinage $V$ de $z_{0}$ et une constante positive $C$ tels que

$$
|Q(z)| \leq C\left(\sum_{i=1}^{m}\left|P_{i}(z)\right|^{2}\right)^{1 / 2}, \quad z \in V .
$$

Le théorème de Briançon-Skoda [BS] (voir aussi [BGVY] ou [BY2] pour une démonstration utilisant le principe du prolongement analytique des distributions $f^{\lambda}$, et par là même rendant explicite le procédé de division), joint au théorème d'extension de Cartan $[\mathrm{H}]$, assure l'existence de polynômes $Q_{1}, \ldots, Q_{m} \in \mathbb{C}\left[z_{1}, \ldots, z_{n}\right]$ tels que

$$
Q^{\inf (n, m)}=Q_{1} P_{1}+\ldots+Q_{m} P_{m} .
$$

On se propose de résoudre explicitement l'équation (1.1) dans $\mathbb{Q}\left[z_{1}\right.$, $\left.\ldots, z_{n}\right]$ lorsque la variété algébrique définie par $P_{1}, \ldots, P_{m}$ est discrète, et ce en donnant un algorithme, ou plutôt une formule, permettant d'expliciter de tels polynômes $Q_{1}, \ldots, Q_{m}$.

De plus, les estimations de degré et de hauteur pour les candidats $Q_{1}, \ldots$, $Q_{m}$ s'approchent au mieux, en ce qui concerne les degrés, des estimations résultant des travaux de Brownawell, Philippon, Kollár ([B1], [CGH], $[\mathrm{P}]$, $[\mathrm{K}]$ ) et, en ce qui concerne les hauteurs, des estimations qui découleraient d'une théorie arithmétique de l'intersection, pour l'instant conjecturelle, telle qu'elle est par exemple proposée par Gillet-Soulé [GS].

Plus précisément, on va montrer qu'étant donnés des polynômes $P_{1}, \ldots$, $P_{m}$ en $n$ variables à coefficients entiers, de degrés respectifs $D=D_{1} \geq$ $\ldots \geq D_{m}$ et de hauteurs logarithmiques (au sens naïf) au plus $h$, dont la variété des zéros est discrète, un polynôme $Q \in \mathbb{Z}\left[z_{1}, \ldots, z_{n}\right]$ de degré $D_{Q}$, et de hauteur logarithmique $h_{Q}$ appartenant à la clôture intégrale 
locale de l'idéal engendré par $P_{1}, \ldots, P_{m}$, il existe alors des polynômes $A_{1}, \ldots, A_{m} \in \mathbb{Z}\left[z_{1}, \ldots, z_{n}\right]$ et un entier naturel non nul $\delta$ tels que

$$
\delta Q^{n}=A_{1} P_{1}+\ldots+A_{m} P_{m}
$$

satisfaisant les estimations suivantes :

$$
\begin{gathered}
\max _{1 \leq i \leq m} \operatorname{deg} A_{i} \leq n^{2}\left(D_{Q}+\Delta\right), \\
\log \delta \leq \kappa(n) D^{a} \Delta^{b}\left(D_{Q}+\Delta\right)^{c}(h+m \log D+D \log D), \\
\max _{1 \leq i \leq m} h\left(A_{i}\right) \leq \kappa(n)\left\{D^{a} \Delta^{b}\left(D_{Q}+\Delta\right)^{c}(h+m \log D+D \log D)+h_{Q}\right\},
\end{gathered}
$$

où $\Delta=D_{1} \ldots D_{n}, a, b, c$ sont des constantes entières absolues (indépendantes de $n$ ) et $\kappa(n)$ une constante ne dépendant que du nombre de variables $n$.

De telles estimations beaucoup moins précises ont été proposées dans [BY1] dans le cas où $P_{1}, \ldots, P_{m}$ n'ont aucun zéro commun. Des estimations concernant le dénominateur $\delta$ suivent des travaux de Philippon, sans que les méthodes proposées dans [P] ou dans [BGS1]-[BGS2] puissent fournir pour l'instant un contrôle des hauteurs des quotients $A_{1}, \ldots, A_{m}$. Il est important de souligner que nous avons ici tenu compte du fait que les degrés $D_{1}, \ldots, D_{m}$ des divers polynômes étaient à priori distincts, ce qui nous autorise à remplacer $D^{n}$ par $D_{1} \ldots D_{n}$ dès que cela s'avère possible.

Je tiens à remercier mon directeur de thèse le Professeur Alain Yger pour son aide constante et ses encouragements et le referee pour ses remarques.

2. Rappels. Soient $\zeta \in \mathbb{C}^{n}, \mathcal{O}_{\zeta}$ l'anneau de germes de fonctions holomorphes en $\zeta$ et $I$ un idéal de $\mathcal{O}_{\zeta}$.

DÉfinition 2.1. Un élément $h \in \mathcal{O}_{\zeta}$ est dit entier (ou intégral) sur $I$ s'il existe un entier strictement positif $k$ tel que $h^{k}+a_{1} h^{k-1}+\ldots+a_{k}=0$, avec $a_{i} \in I^{i}, i=1, \ldots, k$.

On dit alors que $h$ vérifie une relation de dépendance intégrale. On notera l'ensemble des éléments de $\mathcal{O}_{\zeta}$ entiers sur $I$ par $\bar{I}$, et on l'appellera la clôture intégrale de $I$ dans $\mathcal{O}_{\zeta}$; il est bien connu que $\bar{I}$ est un idéal.

En utilisant la résolution des singularités, on peut caractériser la dépendance intégrale par la

Proposition 2.2 [LT]. Soient $I=\left(f_{1}, \ldots, f_{r}\right)$ un idéal de $\mathcal{O}_{\zeta}$ et $h$ un germe de fonctions holomorphes en $\zeta$; alors $h \in \bar{I}$ si, et seulement si, il existe un voisinage $V$ de $\zeta$ et une constante $c>0$ tels que

$$
\forall z \in V, \quad|h(z)| \leq c \max _{1 \leq i \leq r}\left|f_{i}(z)\right| .
$$

La notion de clôture intégrale a été extensivement étudiée par Northcott et Rees [NR], qui ont en particulier introduit la 
DÉFinition 2.3. Soient $J$ et $I$ deux idéaux de $\mathcal{O}_{\zeta}$. On dit que $J$ est une réduction de $I$ si $J \subset I$, et s'il existe $r \in \mathbb{N}^{*}$ tel que $J I^{r}=I^{r+1}$; et on dira qu'une réduction $J$ de $I$ est minimale si aucun idéal strictement inclus dans $J$ n'est une réduction de $I$.

On a alors la proposition suivante :

Proposition 2.4 [NR]. Soit J une réduction de $I$; il existe alors une réduction minimale de I incluse dans $J$.

Compte tenu de cette proposition, on a la

DÉfinition 2.5. Soit $I$ un idéal de $\mathcal{O}_{\zeta}$. On définit l' "analytic spread" $l(I)$ de $I$ comme le nombre d'éléments d'une base minimale d'une réduction minimale de $I$.

Pour un exposé plus complet sur la théorie de réduction des idéaux dans un anneau local, on consultera [NR].

On aura également besoin de la notion de forme nulle telle qu'elle est introduite dans $[\mathrm{NR}]$.

DÉfinition 2.6. Soient $I=\left(u_{1}, \ldots, u_{m}\right)$ un idéal de $\mathcal{O}_{\zeta}$ et $\omega\left(z_{1}, \ldots, z_{m}\right)$ un polynôme homogène de degré $e$ à coefficients dans $\mathcal{O}_{\zeta}$, tel que ses coefficients n'appartiennent pas tous à $m_{\zeta}$, l'idéal maximal de $\mathcal{O}_{\zeta}$. Notons par $\bar{\omega}\left(z_{1}, \ldots, z_{m}\right)$ la forme de degré $e$ correspondante obtenue par passage au quotient modulo $m_{\zeta} ; \bar{\omega}\left(z_{1}, \ldots, z_{m}\right)$ sera dite forme nulle de $I$ lorsque $\omega\left(u_{1}, \ldots, u_{m}\right) \equiv 0 \bmod m_{\zeta} I^{e}$.

On rappelle que la hauteur naïve d'un polynôme $P$ à coefficients entiers, $H(P)$, est le maximum des modules de ses coefficients, et que sa hauteur logarithmique naïve $h(P)$ étant définie par $\log H(P)$.

Enfin, un système de polynômes $P_{1}, \ldots, P_{n} \in \mathbb{C}\left[z_{1}, \ldots, z_{n}\right]$ est dit en position normale si pour tout sous-ensemble $J$ non vide de $\{1, \ldots, n\}$, la variété algébrique $V\left(P_{j}, j \in J\right)=\left\{z \in \mathbb{C}^{n}: P_{j}(z)=0, j \in J\right\}$ est soit vide, soit de codimension cardinal de $J$.

Dans toute la suite $\kappa(n)$ désignera une constante dépendant seulement du nombre de variables $n$, que l'on peut calculer en la suivant pas à pas dans les différentes étapes; on gardera la même notation même si elle change d'une étape à l'autre.

On se placera dans le cas où $n \geq 2$, le nombre de polynômes vérifiant $m>n$. Dans le cas intersection complète $(m=n)$ nous avons une solution du problème d'appartenance à l'idéal $[E]$, qui donne des estimations du même type que celles que nous obtiendrons.

\section{Lemmes préparatoires}

Lemme 3.1. Soient $P_{1}, \ldots, P_{n} \in \mathbb{C}\left[z_{1}, \ldots, z_{n}\right]$, de degrés respectifs au plus $D=D_{1} \geq \ldots \geq D_{n} \geq 3$, et supposons que la variété $V=\left\{z \in \mathbb{C}^{n}\right.$ : 
$\left.P_{1}(z)=\ldots=P_{n}(z)=0\right\}$ soit discrète. Posons $\Delta=D_{1} \ldots D_{n}$. Alors, il existe $n$ combinaisons linéaires $p_{1}, \ldots, p_{n}$ de $P_{1}, \ldots, P_{n}$ en position normale

$$
\begin{gathered}
p_{i}=\sum_{j=1}^{n} \alpha_{i, j} P_{j}, \quad 1 \leq i \leq n, \\
\alpha_{i, j} \in \mathbb{Z}, \quad\left|\alpha_{i, j}\right| \leq(D+1)^{n-1}+\left(\begin{array}{c}
2 n \\
n
\end{array}\right),
\end{gathered}
$$

$n$ formes affines $L_{i} \in \mathbb{Z}\left[z_{1}, \ldots, z_{n}\right], 1 \leq i \leq n$, et une constante $R>0$ telles que

(a) Pour tout sous-ensemble non vide $J$ de $\{1, \ldots, n\}$, pour tout $i$ dans $J$,

$$
V\left(p_{j}, j \in J ; L_{k}, k \notin J ; L_{i}\right)=\emptyset .
$$

(b) Pour tout entier $N \geq 2$, il existe $\gamma_{N}>0$,

$$
\left(\sum_{j=1}^{n}\left|L_{j}^{N \Delta}(z) p_{j}(z)\right|^{2}\right)^{1 / 2} \geq \gamma_{N}\|z\|^{(N-1) \Delta}, \quad\|z\| \geq R .
$$

De plus, si les coefficients des polynômes $P_{1}, \ldots, P_{n}$ sont entiers et de hauteurs logarithmiques au plus $h$, nous pouvons choisir les coefficients des formes $L_{1}, \ldots, L_{n}$ bornés par

$$
\exp \left(\kappa(n) D\left(D_{1} \ldots D_{n-2}\right)(h+D \log D)\right) .
$$

Preuve. Il existe des entiers $\alpha_{i, j}, 1 \leq i, j \leq n,\left|\alpha_{i, j}\right| \leq(D+1)^{n-1}$ tels que les polynômes

$$
p_{i}=\sum_{j=1}^{n} \alpha_{i, j} P_{j}, \quad 1 \leq i \leq n,
$$

soient en position normale ([BY1], Lemme 5.2).

Considérons le système $\left(p_{j}\right)_{j \in J}, J=\left\{i_{1}, \ldots, i_{k}\right\} \subset\{1, \ldots, n\}, 1 \leq$ card $J$ $=k \leq n-1$. Nous allons lui associer un système triangulaire de polynômes $\left(\dot{p}_{J, j}\right)_{j \in J}$; c'est-à-dire tel que deg $\dot{p}_{J, i_{l}} \leq D_{l}, 1 \leq l \leq k$, et que $\dot{p}_{J, j}$ soient des combinaisons linéaires des $p_{j}, j \in J$. Pour simplifier les notations supposons $J=\{1, \ldots, k\}$ et omettons dorénavant l'indice $J$.

On pose $\dot{p}_{1}=p_{1}$. Pour construire $\dot{p}_{2}$, nous éliminons $P_{1}$ entre $p_{2}$ et $p_{1}$; pour cela supposons $\alpha_{1,1} \alpha_{2,1} \neq 0$; alors

$$
\dot{p}_{2}=\alpha_{1,1} p_{2}-\alpha_{2,1} p_{1}=\left|\begin{array}{cc}
\alpha_{1,1} & \alpha_{1,2} \\
\alpha_{2,1} & \alpha_{2,2}
\end{array}\right| P_{2}+\ldots+\left|\begin{array}{cc}
\alpha_{1,1} & \alpha_{1, n} \\
\alpha_{2,1} & \alpha_{2, n}
\end{array}\right| P_{n} .
$$

Pour obtenir $\dot{p}_{3}$, nous éliminons tout d'abord $P_{1}$ entre $p_{3}$ et $p_{1}$, ce qui donnera un polynôme $p_{2}^{(3)}$, puis nous éliminons $P_{2}$ entre $p_{2}^{(3)}$ et $\dot{p}_{2}$; pour cela 
nous supposerons

et nous aurons donc

$$
\alpha_{1,1} \alpha_{1,3}\left|\begin{array}{ll}
\alpha_{1,1} & \alpha_{1,2} \\
\alpha_{2,1} & \alpha_{2,2}
\end{array}\right|\left|\begin{array}{ll}
\alpha_{1,1} & \alpha_{1,2} \\
\alpha_{3,1} & \alpha_{3,2}
\end{array}\right| \neq 0
$$

$$
p_{2}^{(3)}=\alpha_{1,1} p_{3}-\alpha_{3,1} p_{1}=\left|\begin{array}{cc}
\alpha_{1,1} & \alpha_{1,2} \\
\alpha_{3,1} & \alpha_{3,2}
\end{array}\right| P_{2}+\ldots+\left|\begin{array}{cc}
\alpha_{1,1} & \alpha_{1, n} \\
\alpha_{3,1} & \alpha_{3, n}
\end{array}\right| P_{n},
$$

et ainsi

$$
\begin{aligned}
\dot{p}_{3} & =\left|\begin{array}{ll}
\alpha_{1,1} & \alpha_{1,2} \\
\alpha_{2,1} & \alpha_{2,2}
\end{array}\right| p_{2}^{(3)}-\left|\begin{array}{ll}
\alpha_{1,1} & \alpha_{1,2} \\
\alpha_{3,1} & \alpha_{3,2}
\end{array}\right| \dot{p}_{2} \\
& =\alpha_{1,1}\left\{\left|\begin{array}{lll}
\alpha_{1,1} & \alpha_{1,2} & \alpha_{1,3} \\
\alpha_{2,1} & \alpha_{2,2} & \alpha_{2,3} \\
\alpha_{3,1} & \alpha_{3,2} & \alpha_{3,3}
\end{array}\right| P_{3}+\ldots+\left|\begin{array}{lll}
\alpha_{1,1} & \alpha_{1,2} & \alpha_{1, n} \\
\alpha_{2,1} & \alpha_{2,2} & \alpha_{2, n} \\
\alpha_{3,1} & \alpha_{3,2} & \alpha_{3, n}
\end{array}\right| P_{n}\right\}
\end{aligned}
$$

Ce procédé permet de construire $\dot{p}_{1}, \ldots, \dot{p}_{k}$ de proche en proche. Nous montrons par récurrence que si tous les mineurs de la matrice $\left(\alpha_{i, j}\right)_{1 \leq i, j \leq n}$ sont non nuls, $\dot{p}_{l}, 1 \leq l \leq k$, ainsi construits satisfont

$$
\dot{p}_{l}=\Delta_{1} \Delta_{2} \ldots \Delta_{l-2}\left(\Delta_{l} P_{l}+\Delta_{l, \hat{l}, l+1} P_{l+1}+\ldots+\Delta_{l, \hat{l}, n} P_{n}\right), \quad 1 \leq l \leq k,
$$

où

$$
\Delta_{i}=\left|\begin{array}{cccc}
\alpha_{1,1} & \alpha_{1,2} & \ldots & \alpha_{1, i} \\
\alpha_{2,1} & \alpha_{2,2} & \ldots & \alpha_{2, i} \\
\ldots \ldots & \ldots & \ldots & \ldots \\
\alpha_{i, 1} & \alpha_{i, 2} & \ldots & \alpha_{i, i}
\end{array}\right| \quad \text { et } \quad \Delta_{l, \hat{l}, i}=\left|\begin{array}{cccc}
\alpha_{1,1} & \ldots & \alpha_{1, l-1} & \alpha_{1, i} \\
\alpha_{2,1} & \ldots & \alpha_{2, l-1} & \alpha_{2, i} \\
\ldots \ldots & \ldots & \ldots & \ldots \\
\alpha_{l, 1} & \ldots & \alpha_{l, l-1} & \alpha_{l, i}
\end{array}\right|
$$

ceci se fait en utilisant la formule suivante sur les déterminants :

$$
\begin{aligned}
& \left|\begin{array}{cccc}
\alpha_{1,1} & \ldots & \alpha_{1, l-1} & \alpha_{1, l} \\
\ldots \ldots & \ldots & \ldots \ldots \ldots \ldots & \ldots \ldots \\
\alpha_{l-1,1} & \ldots & \alpha_{l-1, l-1} & \alpha_{l-1, l} \\
\alpha_{l, 1} & \ldots & \alpha_{l, l-1} & \alpha_{l, l}
\end{array}\right|\left|\begin{array}{cccc}
\alpha_{1,1} & \ldots & \alpha_{1, l-1} & \alpha_{1, r} \\
\ldots \ldots & \ldots \ldots \ldots \ldots \ldots \ldots \ldots \ldots \ldots \\
\alpha_{l-1,1} & \ldots & \alpha_{l-1, l-1} & \alpha_{l-1, r} \\
\alpha_{l+1,1} & \ldots & \alpha_{l+1, l-1} & \alpha_{l+1, r}
\end{array}\right|
\end{aligned}
$$

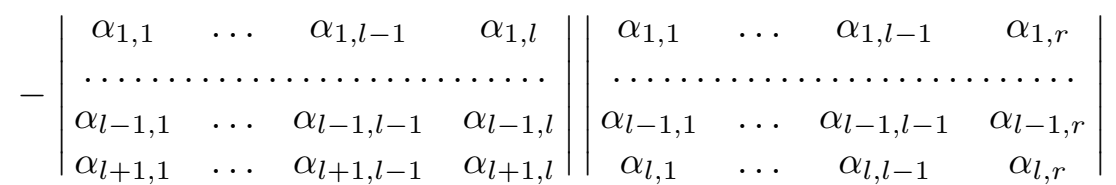

$$
\begin{aligned}
& =\left|\begin{array}{ccc}
\alpha_{1,1} & \ldots & \alpha_{1, l-1} \\
\ldots \ldots \ldots \ldots & \ldots \ldots \ldots \ldots \\
\alpha_{l-1,1} & \ldots & \alpha_{l-1, l-1}
\end{array}\right|\left|\begin{array}{cccc}
\alpha_{1,1} & \ldots & \alpha_{1, l} & \alpha_{1, r} \\
\alpha_{2,1} & \ldots & \alpha_{2, l} & \alpha_{2, r} \\
\ldots \ldots & \ldots & \ldots \ldots \ldots & \ldots \ldots \\
\alpha_{l+1,1} & \ldots & \alpha_{l+1, l} & \alpha_{l+1, r}
\end{array}\right|
\end{aligned}
$$

où les déterminants de la première partie de l'égalité (3.3) sont d'ordre $l$, et ceux de la deuxième partie sont respectivement d'ordre $l-1, l+1$. Il est clair que $\dot{p}_{l} \in\left(p_{1}, \ldots, p_{l}\right), 1 \leq l \leq k$, et que

$$
\dot{p}_{l}=\alpha_{1} p_{1}+\ldots+\alpha_{l-1} p_{l-1}+\Delta_{1} \ldots \Delta_{l-1} p_{l}, \quad \alpha_{1}, \ldots, \alpha_{l-1} \in \mathbb{Z} .
$$


D'après un lemme de zéros immédiat nous pouvons trouver des entiers $\alpha_{i, j}$ dont le module est borné par

$$
(D+1)^{n-1}+\left(\begin{array}{c}
2 n \\
n
\end{array}\right)
$$

tels que les polynômes définis dans (3.2) soient en position normale et tous les mineurs de la matrice $\left(\alpha_{i, j}\right)_{1 \leq i, j \leq n}$ soient non nuls. En faisant ce choix nous voyons que $p_{j}, j \in J$, sont des combinaisons linéaires des $\dot{p}_{J, j}, j \in J$, et vice versa, et ce pour tout $J \subset\{1, \ldots, n\}, 1 \leq$ card $J \leq n-1$. De plus, si les polynômes $P_{1}, \ldots, P_{n}$ sont à coefficients entiers et de hauteurs logarithmiques au plus $h$, les polynômes $p_{i}, 1 \leq i \leq n$, et $\dot{p}_{J, j}, j \in J$, sont aussi à coefficients entiers et de hauteurs logarithmiques bornées par $\kappa(n)(\log D+h)$.

Nous considérons maintenant toutes les familles $\left(\dot{p}_{J, j}\right)_{j \in J}, J \subset\{1, \ldots, n\}$, $1 \leq$ card $J \leq n-1$; leur nombre est $2^{n}-2$. D'après le théorème de normalisation de Noether ([BY1], Remarque 4.6) il existe une matrice inversible à coefficients entiers $A=\left(a_{i, j}\right)_{1 \leq i, j \leq n}$, et deux constantes $\varepsilon>0, K>0$ telles que, pour tout $J \subset\{1, \ldots, n\}, 1 \leq$ card $J=k \leq n-1$, l'ensemble $\mathcal{X}_{J}^{(\varepsilon)}$ défini par

$$
\mathcal{X}_{J}^{(\varepsilon)}=\left\{w \in \mathbb{C}^{n}: \max _{j \in J}\left|\dot{p}_{J, j}(A w)\right|<\varepsilon /(1+\|w\|)^{\Delta}\right\}
$$

est inclus dans

$$
\mathcal{Y}_{k}^{(K)}=\left\{w \in \mathbb{C}^{n}:\left|w_{1}\right|+\ldots+\left|w_{k}\right| \leq K\left(\left|w_{k+1}\right|+\ldots+\left|w_{n}\right|\right)\right\} .
$$

Dans le cas où les polynômes $P_{1}, \ldots, P_{n} \in \mathbb{Z}\left[z_{1}, \ldots, z_{n}\right]$ et $h\left(P_{j}\right) \leq h$, on a les estimations suivantes sur la matrice $A$ et la constante $K$ :

$$
\begin{gathered}
\|A\|=\max _{1 \leq i, j \leq n}\left|a_{i, j}\right| \leq \kappa(n) D^{\kappa(n)}, \\
K \leq \exp \left(\kappa(n) D\left(D_{1} \ldots D_{n-2}\right)(h+D \log D)\right) .
\end{gathered}
$$

Comme les polynômes $p_{1}, \ldots, p_{n}$ sont des combinaisons linéaires des polynômes $P_{1}, \ldots, P_{n}$, et inversement, et que $\operatorname{deg} P_{j} \leq D_{j}, 1 \leq j \leq n$, il existe, d'après les inégalités de Ji-Kollár-Shiffman [JKS], deux constantes $c>0, \varrho>0$ telles que

$$
\max _{1 \leq i \leq n}\left|p_{i}(A w)\right| \geq \frac{c}{(1+\|w\|)^{\Delta}}, \quad\|w\|>\varrho .
$$

Il s'ensuit alors que l'ensemble $\left\{w \in \mathbb{C}^{n}:\|w\|>\varrho\right\}$ peut s'écrire comme l'union disjointe des ensembles

$$
\begin{aligned}
\mathcal{Z}_{J}=\left\{w \in \mathbb{C}^{n}:\|w\|>\varrho,\left|p_{j}(A w)\right|<\frac{c}{(1+\|w\|)^{\Delta}} \text { si } j \in J\right. \\
\text { et } \left.\left|p_{k}(A w)\right| \geq \frac{c}{(1+\|w\|)^{\Delta}} \text { si } k \notin J\right\}
\end{aligned}
$$

où $J \subset\{1, \ldots, n\}, 0 \leq$ card $J \leq n-1$. 
D'autre part, d'après ([BY1], Lemme 5.3) il existe $n$ formes affines $l_{i} \in$ $\mathbb{Z}\left[z_{1}, \ldots, z_{n}\right]$, vérifiant

(a) $h\left(l_{j}\right) \leq \kappa(n) D\left(D_{1} \ldots D_{n-2}\right)(h+D \log D)$ dans le cas où $P_{1}, \ldots, P_{n} \in$ $\mathbb{Z}\left[z_{1}, \ldots, z_{n}\right]$.

(b) Il existe $\delta>0$ tel que pour tout $J \subset\{1, \ldots, n\}, 1 \leq \operatorname{card} J=k \leq n$,

$$
\sum_{j \in J}\left|l_{j}(w)\right| \geq \delta\|w\| \quad \text { si }\left|w_{1}\right|+\ldots+\left|w_{n-k}\right| \leq K\left(\left|w_{n-k+1}\right|+\ldots+\left|w_{n}\right|\right) .
$$

De plus, la construction montre que nous pouvons aussi réaliser, pour tout sous-ensemble $J$ non vide de $\{1, \ldots, n\}$, pour tout $i$ de $J$,

$$
V\left(p_{j}, j \in J ; l_{k} \circ A^{-1}, k \notin J ; l_{i} \circ A^{-1}\right)=\emptyset .
$$

Etant donné un entier $N$, montrons que l'application polynomiale

$$
\left(l_{1}^{N \Delta} p_{1} \circ A, \ldots, l_{n}^{N \Delta} p_{n} \circ A\right)
$$

est propre. En effet, dans (3.4), nous pouvons supposer $c$ arbitrairement petit, ce que nous ferons. Par construction, il existe une constante $d$ telle que pour tout sous-ensemble $J$ de $\{1, \ldots, n\}, 1 \leq \operatorname{card} J \leq n-1$,

$$
\max _{j \in J}\left|\dot{p}_{J, j}(z)\right| \leq d \max _{j \in J}\left|p_{j}(z)\right| .
$$

Soit $w \in \mathcal{Z}_{J}, 1 \leq \operatorname{card} J=k \leq n-1$; alors

$$
\left|\dot{p}_{J, j}(A w)\right| \leq d \max _{j \in J}\left|p_{j}(A w)\right| \leq \frac{d c}{(1+\|w\|)^{\Delta}} \leq \frac{\varepsilon}{(1+\|w\|)^{\Delta}},
$$

donc $w \in \mathcal{X}_{J}^{(\varepsilon)}$, par conséquent $\omega \in \mathcal{Y}_{k}^{(K)}$, ce qui implique $\sum_{j \notin J}\left|l_{j}(w)\right| \geq$ $\delta\|w\|$. Par suite il existe $j_{0} \notin J$ avec $\left|l_{j_{0}}(w)\right| \geq \delta /(n\|w\|)$; ainsi

$$
\left|l_{j_{0}}^{N \Delta}(w) p_{j_{0}}(A w)\right| \geq \delta_{N}\|w\|^{(N-1) \Delta} .
$$

Enfin, les polynômes $p_{1}, \ldots, p_{n}$ et les formes $L_{1}, \ldots, L_{n}$ répondent au lemme 3.1.

Remarque 3.5. Ce lemme utilisé dans la preuve du théorème 5.1 de [BY1] conduit à des bornes pour le problème de Bézout meilleures que celles obtenues dans cet article, dans la mesure où l'on peut séparer les degrés des entrées dans les estimations du dénominateur, des tailles et des degrés des quotients. En effet, nous pouvons résoudre l'identité de Bézout avec les estimations suivantes (voir [BY1], Théorème 5.1) :

$$
\begin{aligned}
\operatorname{deg} q_{j} & \leq n(2 n+1) \Delta, \\
\max \left\{\log \delta, h\left(q_{1}\right), \ldots, h\left(q_{N}\right)\right\} & \leq \kappa(n) D^{4} \Delta^{8}(h+\log N+D \log D),
\end{aligned}
$$

avec $D=\max \left(\operatorname{deg} p_{1}, \ldots, \operatorname{deg} p_{N}\right)$ et $\Delta=\operatorname{deg} p_{1} \ldots \operatorname{deg} p_{n}$. 
Lemme 3.6. Soient $P_{1}, \ldots, P_{m} \in \mathbb{C}\left[z_{1}, \ldots, z_{n}\right]$ de degrés au plus $D$, supposons que la variété $V=\left\{z \in \mathbb{C}^{n}: P_{1}(z)=\ldots=P_{m}(z)=0\right\}$ soit discrète et que $m>n$. Alors, il existe $n$ combinaisons linéaires $q_{i}=\sum_{j=1}^{m} \lambda_{i, j} P_{j}$, $1 \leq i \leq n$, de $P_{1}, \ldots, P_{m}$, à coefficients entiers définissant une variété algébrique discrète et vérifiant

$$
\overline{\left(P_{1}, \ldots, P_{m}\right) \mathcal{O}_{\alpha}}=\overline{\left(q_{1}, \ldots, q_{n}\right) \mathcal{O}_{\alpha}}, \quad \forall \alpha \in V .
$$

De plus, on peut choisir les constantes $\lambda_{i, j}$ de module au plus $D^{n(m-n)}+$ $D^{n-1}$.

Preuve. Soit $\alpha \in V$; notons $I_{\alpha}$ l'idéal de $\mathcal{O}_{\alpha}$ engendré par $P_{1}, \ldots, P_{m}$, qui est $m_{\alpha}$-primaire. Alors, d'après ([NR], Théorème 1 , page 154) l' 'analytic spread" de $I_{\alpha}$ est égal à $n$. Ceci nous assure l'existence de $n$ combinaisons linéaires $g_{1}, \ldots, g_{n}$ de $P_{1}, \ldots, P_{m}$ qui engendrent une réduction minimale de $I_{\alpha}$ (on peut choisir les mêmes combinaisons pour tout $\alpha \in V$, et supposer la variété $V\left(g_{1}, \ldots, g_{n}\right)$ discrète). Nous aurons donc

$$
\forall \alpha \in V, \quad \overline{\left(g_{1}, \ldots, g_{n}\right) \mathcal{O}_{\alpha}}=\overline{\left(P_{1}, \ldots, P_{m}\right) \mathcal{O}_{\alpha}} .
$$

Complétons $\left(g_{1}, \ldots, g_{n}\right)$ par $g_{n+1}, \ldots, g_{m}$ pour que $\left(g_{1}, \ldots, g_{m}\right)=$ $\left(P_{1}, \ldots, P_{m}\right)$ dans $\mathbb{C}\left[z_{1}, \ldots, z_{n}\right]$. Puisque pour tout $n+1 \leq k \leq m, g_{k} \in$ $\overline{\left(g_{1}, \ldots, g_{n}\right) \mathcal{O}_{\alpha}}$, il s'ensuit que $g_{k}$ vérifie une relation intégrale explicite sur $\left(g_{1}, \ldots, g_{n}\right) \mathcal{O}_{\alpha}$ de degré $e_{\alpha}$ [Hé] :

$$
g_{k}^{e_{\alpha}}+a_{1}^{(k)} g_{k}^{e_{\alpha}-1}+\ldots+a_{i}^{(k)} g_{k}^{e_{\alpha}-i}+\ldots+a_{e_{\alpha}}^{(k)}=0
$$

où

$$
e_{\alpha}=\operatorname{dim}_{\mathbb{C}}\left(\mathcal{O}_{\alpha} /\left(g_{1}, \ldots, g_{n}\right) \mathcal{O}_{\alpha}\right)
$$

est la multiplicité de l'idéal $\left(g_{1}, \ldots, g_{n}\right) \mathcal{O}_{\alpha}$ et

$$
a_{i}^{(k)}=\sum_{j_{1}+\ldots+j_{n}=i} h_{i, j}^{(k)} g_{1}^{j_{1}} \ldots g_{n}^{j_{n}}, \quad h_{i, j}^{(k)} \in \mathcal{O}_{\alpha} .
$$

Nous avons donc, pour tout $\alpha$ dans $V$,

$$
g_{k}^{e_{\alpha}}+\sum_{i=1}^{e_{\alpha}} \sum_{j_{1}+\ldots+j_{n}=i} h_{i, j}^{(k)}(\alpha) g_{1}^{j_{1}} \ldots g_{n}^{j_{n}} g_{k}^{e_{\alpha}-i} \in m_{\alpha} I_{\alpha}^{e_{\alpha}} .
$$

Considérons les $m-n$ polynômes homogènes non nuls, à coefficients dans $\mathbb{C}$, de degrés $e=\sum_{\alpha \in V} e_{\alpha} \leq D^{n}([\mathrm{GH}],[\mathrm{T}])$, s'annulant en $\left(g_{1}, \ldots, g_{m}\right)$ modulo $m_{\alpha} I_{\alpha}^{e_{\alpha}}$ pour tout $\alpha$ de $V$, définis par

$$
\begin{array}{r}
\omega_{k}\left(z_{1}, \ldots, z_{m}\right)=\prod_{\alpha \in V}\left(z_{k}^{e_{\alpha}}+\sum_{i=1}^{e_{\alpha}} \sum_{j_{1}+\ldots+j_{n}=i} h_{i, j}^{(k)}(\alpha) z_{1}^{j_{1}} \ldots z_{n}^{j_{n}} z_{k}^{e_{\alpha}-i}\right), \\
n+1 \leq k \leq m .
\end{array}
$$


Les formes $\omega_{n+1}, \ldots, \omega_{m}$, sont des formes nulles de $I_{\alpha}$ (au sens de la définition 2.6), et ce pour tout $\alpha$ dans $V$. Cette construction nous a été suggérée par Michel Hickel.

Pour trouver des polynômes $q_{1}, \ldots, q_{n}$ satisfaisant (3.7), il est suffisant d'après ([NR], Lemme 1 , page 152) de trouver des entiers $\lambda_{i, j}, 1 \leq i \leq n, 1 \leq$ $j \leq m$, vérifiant

$$
\begin{aligned}
\left\{z \in \mathbb{C}^{n}: \sum_{j=1}^{m} \lambda_{1, j} z_{j}=\ldots=\sum_{j=1}^{m} \lambda_{n, j} z_{j}=\varphi(z)=0, \forall \varphi \in \mathcal{I}_{\alpha}\right\} & =\{0\}, \\
& \forall \alpha \in V,
\end{aligned}
$$

où $\mathcal{I}_{\alpha}$ est l'idéal homogène engendré par les formes nulles de $I_{\alpha}$. Il suffit pour cela que les entiers $\lambda_{i, j}$ satisfassent

$\left\{z \in \mathbb{C}^{n}: \sum_{j=1}^{m} \lambda_{1, j} z_{j}=\ldots=\sum_{j=1}^{m} \lambda_{n, j} z_{j}=\omega_{n+1}(z)=\ldots=\omega_{m}(z)=0\right\}=\{0\}$,

ce qui est équivalent au fait que le résultant de ces $m$ polynômes n'est pas nul [V]. Or, d'après un lemme immédiat de zéros, on peut trouver des entiers $\lambda_{i, j}$ qui réalisent ceci et dont le module est borné par $D^{n(m-n)}+D^{n-1}$.

Finalement, les polynômes $q_{i}=\sum_{j=1}^{m} \lambda_{i, j} P_{j}, 1 \leq i \leq n$, répondent au lemme 3.6.

Corollaire 3.8. Soient $P_{1}, \ldots, P_{m} \in \mathbb{Z}\left[z_{1}, \ldots, z_{n}\right]$ de degrés respectifs $D=D_{1} \geq \ldots \geq D_{m} \geq 3$ et de hauteurs logarithmiques au plus $h$. On suppose que la variété $V=V\left(P_{1}, \ldots, P_{m}\right)$ est discrète et que $m>n$. Posons $\Delta=D_{1} \ldots D_{n}$. Alors, il existe des entiers $\gamma_{i, j}, 1 \leq i \leq n, 1 \leq j \leq m$, de module au plus $\exp (\kappa(n) m \log D)$, ainsi que $n$ formes affines $L_{1}, \ldots, L_{n} \in$ $\mathbb{Z}\left[z_{1}, \ldots, z_{n}\right]$ et une constante $R>0$ tels que : male.

( $\alpha$ Les polynômes $p_{i}=\sum_{j=1}^{m} \gamma_{i, j} P_{j}, 1 \leq i \leq n$, sont en position nor-

(ß) $h\left(L_{i}\right) \leq \kappa(n) D\left(D_{1} \ldots D_{n-2}\right)(h+m \log D+D \log D), 1 \leq i \leq n$.

$(\gamma)$ Pour tout sous-ensemble non vide $J$ de $\{1, \ldots, n\}$, et pour tout $i$ dans $J$,

$$
V\left(p_{j}, j \in J ; L_{k}, k \notin J ; L_{i}\right)=\emptyset .
$$

(ठ) Pour tout entier $N \geq 2$, il existe $\gamma_{N}>0$ tel que

$$
\left(\sum_{i=1}^{n}\left|L_{i}^{N \Delta}(z) p_{i}(z)\right|^{2}\right)^{1 / 2} \geq \gamma_{N}\|z\|^{(N-1) \Delta}, \quad\|z\| \geq R .
$$

(є) Pour tout $\alpha \in V, \overline{\left(P_{1}, \ldots, P_{m}\right) \mathcal{O}_{\alpha}}=\overline{\left(p_{1}, \ldots, p_{n}\right) \mathcal{O}_{\alpha}}$.

Preuve. En utilisant le procédé décrit dans la preuve du lemme 3.1, nous pouvons construire $n$ combinaisons linéaires $q_{1}, \ldots, q_{n}$ de $P_{1}, \ldots, P_{m}$, 
avec $\operatorname{deg} q_{i} \leq D_{i}$ et $h\left(q_{i}\right) \leq \kappa(n)(h+m \log D)$, qui vérifient, pour tout $\alpha \in V$,

$$
\overline{\left(P_{1}, \ldots, P_{m}\right) \mathcal{O}_{\alpha}}=\overline{\left(q_{1}, \ldots, q_{n}\right) \mathcal{O}_{\alpha}} .
$$

Le corollaire 3.8 découle du lemme 3.1 appliqué aux polynômes $q_{1}, \ldots, q_{n}$.

R e m a r qu e 3.11. Dans le cas particulier du problème de Bézout, l'estimation des hauteurs des formes $L_{1}, \ldots, L_{n}$ satisfaisant le corollaire 3.8 est

$$
\max _{1 \leq i \leq n} h\left(L_{i}\right) \leq \kappa(n) D\left(D_{1} \ldots D_{n-2}\right)(h+\log m+D \log D) .
$$

4. Formules de division. Supposons donnés $m$ polynômes $P_{1}, \ldots, P_{m}$ vérifiant les hypothèses du corollaire 3.8 , auxquels nous attacherons un choix de $n$ combinaisons linéaires $p_{1}, \ldots, p_{n}$ de $P_{1}, \ldots, P_{m}$ et de $n$ formes affines $L_{1}, \ldots, L_{n}$. Considérons un polynôme $Q$ de degré $D_{Q}$, qui est localement dans la clôture intégrale de $\left(P_{1}, \ldots, P_{m}\right)$.

Fixons $N=4 n+n\left[D_{Q} / \Delta\right]$, où $\left[D_{Q} / \Delta\right]$ est la partie entière de $D_{Q} / \Delta$ et $M=N \Delta$.

Notons $\left(f_{j, k}\right)_{1 \leq j, k \leq n}$ une matrice de polynômes en $2 n$ variables réalisant un système de diviseurs de Hefer pour les polynômes $L_{j}^{M} p_{j}, 1 \leq j \leq n$, soit :

$$
\forall z, \zeta \in \mathbb{C}^{n}, \quad L_{j}^{M}(z) p_{j}(z)-L_{j}^{M}(\zeta) p_{j}(\zeta)=\sum_{k=1}^{n} f_{j, k}(z, \zeta)\left(z_{k}-\zeta_{k}\right),
$$

$$
1 \leq j \leq n
$$

par exemple, on peut prendre

$$
\begin{aligned}
& f_{j, k}(z, \zeta) \\
= & \frac{L_{j}^{M} p_{j}\left(\zeta_{1}, \ldots, \zeta_{k-1}, z_{k}, \ldots, z_{n}\right)-L_{j}^{M} p_{j}\left(\zeta_{1}, \ldots, \zeta_{k}, z_{k+1}, \ldots, z_{n}\right)}{z_{k}-\zeta_{k}} .
\end{aligned}
$$

Notons $\left(P_{j, k}\right)_{1 \leq k \leq n}$ un système de diviseurs de Hefer pour $P_{j}, 1 \leq j \leq m$. Nous introduisons les déterminants

$$
H_{j}(z, \zeta)=\left|\begin{array}{cccc}
f_{1,1}(z, \zeta) & \ldots & f_{n, 1}(z, \zeta) & P_{j, 1}(z, \zeta) \\
\ldots \ldots \ldots \ldots \ldots \ldots \ldots \ldots \ldots \ldots \ldots \ldots \ldots \ldots \ldots & \ldots \ldots \ldots \\
f_{1, n}(z, \zeta) & \ldots & f_{n, n}(z, \zeta) & P_{j, n}(z, \zeta) \\
L_{1}^{M}(z) p_{1}(z) & \ldots & L_{n}^{M}(z) p_{n}(z) & P_{j}(z)
\end{array}\right|, \quad 1 \leq j \leq m .
$$

Soient $\alpha \in\left\{z \in \mathbb{C}^{n}: L_{1} p_{1}(z)=\ldots=L_{n} p_{n}(z)=0\right\}$ et $R$ une fraction rationnelle sans pôles en $\alpha$. Le résidu local en $\alpha$ de la forme différentielle $R d \zeta$ associé à l'application polynomiale $L^{M} p=\left(L_{1}^{M} p_{1}, \ldots, L_{n}^{M} p_{n}\right)$ est noté $\left\langle\bar{\partial}\left(1 / L^{M} p\right), R d \zeta\right\rangle_{\alpha}([\mathrm{GH}],[\mathrm{T}])$. 
THÉORÈME 4.2. Sous les hypothèses et notations précédentes, nous avons la formule de division suivante:

$$
Q^{n}(z)=\sum_{\substack{\alpha \in \mathbb{C}^{n}: L_{i} p_{i}(\alpha)=0 \\ \Sigma_{i=1}^{m}\left|P_{i}(\alpha)\right|>0}}\left\langle\bar{\partial}\left(1 / L^{M} p\right), \frac{Q^{n}}{\sum_{k=1}^{m} \beta_{k} P_{k}} \sum_{j=1}^{m} \beta_{j} H_{j}(z, \zeta) d \zeta\right\rangle_{\alpha}
$$

où $\beta_{1}, \ldots, \beta_{m}$ sont des constantes entières de modules au plus $(D+1)^{n}$ choisies de manière à ce que $\sum_{k=1}^{m} \beta_{k} P_{k}$ ne s'annule pas sur $\left\{z \in \mathbb{C}^{n}\right.$ : $\left.L_{i} p_{i}(z)=0,1 \leq i \leq n, \sum_{k=1}^{m}\left|P_{k}(z)\right|>0\right\}$.

Preuve. Nous appliquerons aux polynômes $L_{1}^{M} p_{1}, \ldots, L_{n}^{M} p_{n}, P_{1}, \ldots$, $P_{m}$, que nous noterons respectivement $f_{1}, \ldots, f_{n+m}$, la formule de division établie dans ([BY1], Théorème 3.1) applicable ici du fait des inégalités (3.10). Pour cela nous allons écrire $Q^{n}=\sum_{i=1}^{n+m} u_{i} f_{i}$ dans un voisinage de $V\left(L_{1} p_{1}, \ldots, L_{n} p_{n}\right)$, avec $u_{i}, 1 \leq i \leq n+m$, des fonctions holomorphes.

Autour des zéros communs à $L_{1} p_{1}, \ldots, L_{n} p_{n}$ qui ne sont pas zéros communs de $P_{1}, \ldots, P_{m}$ nous écrirons

$$
Q^{n}=\frac{\beta_{1} Q^{n}}{\sum_{k=1}^{m} \beta_{k} P_{k}} P_{1}+\ldots+\frac{\beta_{m} Q^{n}}{\sum_{k=1}^{m} \beta_{k} P_{k}} P_{m},
$$

$\beta_{k}, 1 \leq k \leq m$, étant des entiers tels que $\sum_{k=1}^{m} \beta_{k} P_{k}$ ne s'annule en aucun de ces points. En effet, on peut trouver de tels entiers dont le module est borné par $(D+1)^{n}$.

Autour d'un zéro $\alpha$ commun à $P_{1}, \ldots, P_{m}$, le théorème de BriançonSkoda et le corollaire 3.8 assurent que $Q^{n} \in\left(p_{1}, \ldots, p_{n}\right) \mathcal{O}_{\alpha}$. D'après le choix (3.9) des formes affines $L_{1}, \ldots, L_{n}$, nous avons aussi

$$
Q^{n} \in\left(L_{1}^{M} p_{1}, \ldots, L_{n}^{M} p_{n}\right) \mathcal{O}_{\alpha} .
$$

La formule de division mentionnée au début de la preuve donne

$$
\begin{aligned}
Q^{n} & =\left\langle\bar{\partial}\left(1 / L^{M} p\right), \sum_{j=1}^{n+m} u_{j}\left|\begin{array}{cccc}
f_{1,1}(z, \zeta) & \ldots & f_{n, 1}(z, \zeta) & f_{j, 1}(z, \zeta) \\
\ldots \ldots \ldots \ldots \ldots \ldots \ldots \ldots \ldots \ldots \ldots \ldots \\
f_{1, n}(z, \zeta) & \ldots & f_{n, n}(z, \zeta) & f_{j, n}(z, \zeta) \\
f_{1}(z)-f_{1}(\zeta) & \ldots & f_{n}(z)-f_{n}(\zeta) & f_{j}(z)
\end{array}\right| d \zeta\right\rangle \\
& =\sum_{\substack{\alpha \in \mathbb{C}^{n}: L_{i} p_{i}(\alpha)=0 \\
\Sigma_{i=1}^{m}\left|P_{i}(\alpha)\right|>0}}\left\langle\bar{\partial}\left(1 / L^{M} p\right), \frac{Q^{n}}{\sum_{k=1}^{m} \beta_{k} P_{k}} \sum_{j=1}^{m} \beta_{j} H_{j}(z, \zeta) d \zeta\right\rangle_{\alpha} . \cdot
\end{aligned}
$$

Re marque 4.4. La construction de fonctions holomorphes résolvant le problème de la division au voisinage de $V\left(L_{1} p_{1}, \ldots, L_{n} p_{n}\right)$ dans la preuve du théorème 4.2 ne nécessite pas, comme dans ([BY2], Théorème 2), le recours à la formule de Weil; d'autre part, dans la formule de division (4.3), les zéros des polynômes originaux ne jouent pas de rôle, contrairement à ce qui se passe dans [BY2]. 
ThÉORÈme 4.5. Soient $P_{1}, \ldots, P_{m} \in \mathbb{Z}\left[z_{1}, \ldots, z_{n}\right]$ de degrés respectifs au plus $D=D_{1} \geq \ldots \geq D_{m} \geq 3$ et de hauteurs logarithmiques au plus $h$. On suppose que la variété algébrique définie par $P_{1}, \ldots, P_{m}$ est discrète et que $m>n$. Posons $\Delta=D_{1} \ldots D_{n}$; alors pour tout $Q \in \mathbb{Z}\left[z_{1}, \ldots, z_{n}\right]$ de degré $D_{Q}$ et de hauteur logarithmique $h_{Q}$ qui est localement dans la clôture intégrale de l'idéal engendré par $P_{1}, \ldots, P_{m}$, il existe des polynômes $A_{1}, \ldots, A_{m} \in \mathbb{Z}\left[z_{1}, \ldots, z_{n}\right]$ et un entier $\delta$ strictement positif tels que

$$
\delta Q^{n}=A_{1} P_{1}+\ldots+A_{m} P_{m}
$$

satisfaisant les estimations

$$
\begin{gathered}
\max _{1 \leq i \leq m} \operatorname{deg} A_{i} \leq n^{2} D_{Q}+n(4 n+1) \Delta, \\
\log \delta \leq \kappa(n) D^{4} \Delta^{6}\left(D_{Q}+\Delta\right)^{2}(h+m \log D+D \log D), \\
\max _{1 \leq i \leq m} h\left(A_{i}\right) \leq \kappa(n)\left\{D^{4} \Delta^{5}\left(D_{Q}+\Delta\right)^{3}(h+m \log D+D \log D)+h_{Q}\right\} .
\end{gathered}
$$

Remarque 4.6. On peut remplacer $\mathbb{Z}$ par l'anneau des entiers d'un corps de nombres dans le théorème 4.5. Dans ce cas, la constante $\kappa(n)$ qui figure dans les estimations dépendra, en plus du nombre de variables, du degré du corps de nombres considéré.

Preuve. En développant le déterminant qui apparaît dans la formule de division (4.3) selon la dernière ligne, nous aurons

$$
Q^{n}(z)=\sum_{k=1}^{m} P_{k}(z) Q_{k}(z)
$$

avec $Q_{k}(z)$ égal à

$$
\begin{aligned}
\sum_{\substack{\alpha \in \mathbb{C}^{n}: L_{i} p_{i}(\alpha)=0 \\
\Sigma_{i=1}^{m}\left|P_{i}(\alpha)\right|>0}}\left\langle\bar{\partial}\left(1 / L^{M} p\right),\right. \\
\left.\quad \frac{Q^{n}(\zeta)}{R(\zeta)}\left(\beta_{k} H(z, \zeta)+\sum_{\substack{1 \leq l \leq n \\
1 \leq j \leq m}} \beta_{j} \gamma_{l, k} L_{l}^{M}(z) H_{j, \hat{l}}(z, \zeta)\right) d \zeta\right\rangle_{\alpha}
\end{aligned}
$$

où $R(\zeta)=\sum_{k=1}^{m} \beta_{k} P_{k}(\zeta), H(z, \zeta)$ s'obtient à partir de $H_{j}(z, \zeta)$ en éliminant la dernière ligne et la dernière colonne et $H_{j, \hat{l}}(z, \zeta)$ en éliminant la dernière ligne et la $l$-ème colonne. Il est clair que $Q_{1}, \ldots, Q_{m}$ sont des polynômes en les variables $z_{1}, \ldots, z_{n}$ de degrés au plus $n^{2} D_{Q}+n(4 n+1) \Delta$, et à coefficients algébriques. En suivant [BY1], nous nous proposons de chercher un dénominateur commun aux coefficients des polynômes $Q_{1}, \ldots, Q_{m}$ et d'estimer la taille de ces coefficients.

Tout d'abord nous rappelons quelques définitions et propriétés des nombres algébriques [W]. Soit $\alpha$ un nombre algébrique; on note $|\bar{\alpha}|=\max \{|\beta|: \beta$ est un $\mathbb{Q}$-conjugué de $\alpha$ \}. Le dénominateur de $\alpha$, $\operatorname{den}(\alpha)$, est le plus petit 
entier strictement positif $d$ tel que $d \alpha$ est un entier algébrique. La taille de $\alpha, \mathrm{t}(\alpha)$, est définie par $\max (\log \operatorname{den}(\alpha), \log |\bar{\alpha}|)$. On a

$$
\log \operatorname{den}\left(\frac{1}{\alpha}\right) \leq 2 \mathrm{t}(\alpha) \operatorname{deg}(\alpha)
$$

Soient $p \in \mathbb{Z}\left[z_{1}, \ldots, z_{n}\right], r_{1}, \ldots, r_{n}$ des entiers tels que $\operatorname{deg} p_{z_{i}} \leq r_{i}$ et $\alpha_{1}, \ldots, \alpha_{n}$ des nombres algébriques. Alors $p\left(\alpha_{1}, \ldots, \alpha_{n}\right)$ est un nombre algébrique; son dénominateur

$$
\operatorname{den}\left(p\left(\alpha_{1}, \ldots, \alpha_{n}\right)\right) \text { divise } \prod_{i=1}^{n}\left(\operatorname{den}\left(\alpha_{i}\right)\right)^{r_{i}}
$$

et sa taille satisfait

$$
\mathrm{t}\left(p\left(\alpha_{1}, \ldots, \alpha_{n}\right)\right) \leq h(p)+\sum_{i=1}^{n}\left(r_{i} \mathrm{t}\left(\alpha_{i}\right)+\log \left(r_{i}+1\right)\right) .
$$

Les coefficients des polynômes $Q_{i}, 1 \leq i \leq m$, sont des combinaisons linéaires entières de nombres algébriques de la forme

$$
\sum_{\substack{\alpha \in \mathbb{C}^{n}: L_{i} p_{i}(\alpha)=0 \\ \Sigma_{i=1}^{m}\left|P_{i}(\alpha)\right|>0}}\left\langle\bar{\partial}\left(1 / L^{M} p\right), \frac{\zeta^{k}}{R(\zeta)} d \zeta\right\rangle_{\alpha}, \quad|k| \leq \kappa(n)\left(D_{Q}+\Delta\right),
$$

que nous pouvons écrire, d'après (3.9), sous la forme

$$
\begin{aligned}
& \sum_{l=0}^{n} \sum_{\begin{array}{c}
J \subset\{1, \ldots, n\} \\
\operatorname{card} J=l
\end{array}} \sum_{\begin{array}{c}
\alpha \in \mathbb{C}^{n}: p_{i}(\alpha)=0, i \in J \\
L_{j}(\alpha)=0, j \notin J \\
\Sigma_{l=1}^{m}\left|P_{l}(\alpha)\right|>0
\end{array}} \\
&\left\langle\left(\bigwedge_{i \in J} \bar{\partial}\left(1 / p_{i}\right) \bigwedge_{j \notin J} \bar{\partial}\left(1 / L_{j}^{M}\right)\right)^{\prime}, \frac{\zeta^{k}}{\widetilde{R}(\zeta)} d \zeta\right\rangle_{\alpha}
\end{aligned}
$$

avec $\widetilde{R}(\zeta)=R(\zeta)\left(\prod_{i \in J} L_{i}^{M}(\zeta)\right)\left(\prod_{j \notin J} p_{j}\right)$. Le prime dans le premier membre du crochet de (4.11) signifie que l'on prend le résidu local en $\alpha$, après avoir réarrangé les indices dans l'ordre strictement croissant.

La construction donnée dans la preuve du lemme 3.1 assure l'existence, pour tout sous-ensemble non vide $J=\left\{i_{1}, \ldots, i_{l}\right\}$ de $\{1, \ldots, n\}, 1 \leq$ card $J$ $=l \leq n$, de $l$ polynômes $\dot{p}_{i_{1}}, \ldots, \dot{p}_{i_{l}}, \dot{p}_{i_{j}}=\sum_{k=1}^{l} \alpha_{i_{j}, k} p_{i_{k}}$, où $\alpha_{i_{j}, k}, 1 \leq k \leq$ $l$, sont des entiers et $\operatorname{deg} p_{i_{j}} \leq D_{j}$. En utilisant la loi de transformation du résidu ([GH], $[\mathrm{T}]$ ), l'expression (4.11) devient une somme de nombres algébriques de la forme

$$
\begin{aligned}
c_{J}\left\langle\left(\bigwedge_{i \in J} \bar{\partial}\left(1 / \dot{p}_{i}\right) \bigwedge_{j \notin J} \bar{\partial}\left(1 / L_{j}^{M}\right)\right)^{\prime}, \frac{\zeta^{k}}{\widetilde{R}(\zeta)} d \zeta\right\rangle_{\alpha}, \\
c_{J} \in \mathbb{Z},\left|c_{J}\right| \leq \exp (\kappa(n) m \log D) .
\end{aligned}
$$


En écrivant $1 / \dot{p}_{i}=\dot{p}_{i}^{M-1} / \dot{p}_{i}^{M}$, nous nous ramenons à la recherche d'un dénominateur commun aux nombres algébriques

$$
\begin{aligned}
\left\langle\left(\bigwedge_{i \in J} \bar{\partial}\left(1 / \dot{p}_{i}^{M}\right) \bigwedge_{j \notin J} \bar{\partial}\left(1 / L_{j}^{M}\right)\right)^{\prime}, \frac{\zeta^{k}}{\widetilde{R}(\zeta)} d \zeta\right\rangle_{\alpha}, & \\
& |k| \leq \kappa(n) D\left(D_{Q}+\Delta\right) .
\end{aligned}
$$

Posons $\Phi=\left(\Phi_{1}, \ldots, \Phi_{n}\right)=\left(\dot{p}_{i}, i \in J ; L_{j}, j \notin J\right)^{\prime}$. Pour calculer explicitement les expressions (4.13), nous combinerons la théorie de l'élimination et la théorie du résidu de Grothendieck. En effet, les polynômes $\Phi_{1}, \ldots, \Phi_{n}$ sont de degrés respectifs au plus $D_{1}, \ldots, D_{n}$ et de hauteurs logarithmiques bornées par $\kappa(n) D \Delta(h+m \log D+D \log D)$; le lemme 4.3 de [BY1] appliqué à $T=z_{j}, X=\left(z_{1}, \ldots, \widehat{z}_{j}, \ldots, z_{n}\right), 1 \leq j \leq n$, implique l'existence de polynômes $B_{1}, \ldots, B_{n}$, où $B_{j}$ dépend seulement de la variable $z_{j}$, appartenant à l'idéal de $\mathbb{Z}\left[z_{1}, \ldots, z_{n}\right]$ engendré par $\Phi_{1}, \ldots, \Phi_{n}$, satisfaisant :

$$
\begin{gathered}
\operatorname{deg} B_{j} \leq \kappa(n) D \Delta, \\
h\left(B_{j}\right) \leq \kappa(n) D^{2} \Delta^{2}(h+m \log D+D \log D) .
\end{gathered}
$$

Malheureusement, à ce stade, nous n'avons pas d'information sur les degrés des quotients $B_{j, k}, 1 \leq j, k \leq n$, tels que $B_{j}=\sum_{k=1}^{n} B_{j, k} \Phi_{k}$. Pour remédier à ceci nous utiliserons l'astuce de Rabinowitsch.

Fixons $j \in\{1, \ldots, n\}$ et soit $T$ un entier tel que $2 T \geq D_{1}, \operatorname{deg} B_{j} \leq T \leq$ $\kappa(n) D \Delta$, et considérons les polynômes de $\mathbb{Z}\left[z_{0}, \ldots, z_{n}\right]$

$$
\Phi_{0}\left(z_{0}, \ldots, z_{n}\right)=1-z_{0}^{T} B_{j}(z), \quad \Phi_{1}, \quad \ldots, \quad \Phi_{n},
$$

de degrés respectifs au plus $2 T, D_{1}, \ldots, D_{n}$, et de hauteurs logarithmiques bornées par $\kappa(n) D^{2} \Delta^{2}(h+m \log D+D \log D)$. Ces polynômes n'ont pas de zéros communs dans $\mathbb{C}^{n}$. Il existe alors $S_{0}, \ldots, S_{n} \in \mathbb{Z}\left[z_{0}, \ldots, z_{n}\right]$ et $a_{j} \in \mathbb{N}^{*}$ tels que $([\mathrm{P}]$, Théorème 4$)$

$$
a_{j}=S_{0}\left(1-z_{0}^{T} B_{j}\right)+S_{1} \Phi_{1}+\ldots+S_{n} \Phi_{n}
$$

avec

$$
\begin{gathered}
\operatorname{deg}\left(S_{i} \Phi_{i}\right) \leq(n+4) 2 T \Delta, \\
\log a_{j} \leq \kappa(n) D^{3} \Delta^{4}(h+m \log D+D \log D) .
\end{gathered}
$$

En décomposant $S_{i}, 0 \leq i \leq n$, sous la forme

$$
S_{i}\left(z_{0}, \ldots, z_{n}\right)=\sum_{k=0}^{T-1} S_{i, k}\left(z_{0}^{T}, z_{1}, \ldots, z_{n}\right) z_{0}^{k},
$$

l'identité (4.14) devient

$$
a_{j}=S_{0,0}\left(z_{0}^{T}, z\right)\left(1-z_{0}^{T} B_{j}(z)\right)+\ldots+S_{n, 0}\left(z_{0}^{T}, z\right) \Phi_{n}(z) .
$$


En remplaçant $z_{0}^{T}$ par $X$ dans (4.14), nous avons

$$
\operatorname{deg}_{X} S_{i, 0} \leq 2(n+4) \Delta .
$$

Et en posant $X=1 / B_{j}$, on définit

$$
b_{j}=a_{j} B_{j}^{\gamma}=\sum_{l=1}^{n} b_{j, l} \Phi_{l}
$$

où $\gamma=2(n+4) \Delta$, et $b_{j, l}, 1 \leq j, l \leq n$, sont des polynômes à coefficients entiers de degrés au plus $\kappa(n) D \Delta^{2}$. Par suite,

$b_{j}^{n M}=\sum_{l=1}^{n} b_{j, l}^{(M)} \Phi_{l}^{M}, \quad b_{j, l}^{(M)} \in \mathbb{Z}\left[z_{1}, \ldots, z_{n}\right], \operatorname{deg} b_{j, l}^{(M)} \leq \kappa(n) D \Delta^{2}\left(D_{Q}+\Delta\right)$.

En appliquant la loi de transformation du résidu ([GH], [T]), nous trouvons

$$
\left\langle\bar{\partial}\left(1 / \Phi^{M}\right), \frac{\zeta^{k}}{\widetilde{R}(\zeta)} d \zeta\right\rangle_{\alpha}=\left\langle\bar{\partial}\left(1 / b^{n M}\right), \frac{\zeta^{k} \operatorname{det}\left(b_{j, l}^{(M)}\right)_{1 \leq j, l \leq n}}{\widetilde{R}(\zeta)} d \zeta\right\rangle_{\alpha} .
$$

Puisque nous nous intéressons, pour le moment, seulement aux dénominateurs, nous sommes ramenés à la recherche d'un dénominateur commun aux nombres algébriques

$$
\left\langle\bar{\partial}\left(1 / b^{n M}\right), \frac{\zeta^{k}}{\widetilde{R}(\zeta)} d \zeta\right\rangle_{\alpha}, \quad|k| \leq \kappa(n) D \Delta^{2}\left(D_{Q}+\Delta\right) .
$$

L'avantage des quantités (4.15) est le fait de pouvoir les calculer par itération de la formule du résidu usuelle à une seule variable.

Soit $\nu_{j}$ la multiplicité de $\alpha_{j}$ comme zéro de $B_{j}$; alors

$$
B_{j}\left(z_{j}\right)=\left(z_{j}-\alpha_{j}\right)^{\nu_{j}} \theta_{j}\left(z_{j}\right), \quad \theta_{j} \in \mathbb{Z}\left[\alpha_{j}\right]\left[z_{j}\right], \theta_{j}\left(\alpha_{j}\right) \neq 0 .
$$

Alors,

$$
\left\langle\bar{\partial}\left(1 / b^{n M}\right), \frac{\zeta^{k}}{\widetilde{R}(\zeta)} d \zeta\right\rangle_{\alpha}=\frac{1}{\left(a_{1} \ldots a_{n}\right)^{n M}} \frac{1}{\mathcal{M} !}\left(\frac{\partial^{|\mathcal{M}|} \Theta_{k}}{\partial z^{\mathcal{M}}}\right)(\alpha),
$$

où

$\mathcal{M}=\left(n M \gamma \nu_{1}-1, \ldots, n M \gamma \nu_{n}-1\right)$ et $\Theta_{k}(\zeta)=\frac{1}{\left(\theta_{1}\left(\zeta_{1}\right) \ldots \theta_{n}\left(\zeta_{n}\right)\right)^{n M \gamma}} \frac{\zeta^{k}}{\widetilde{R}(\zeta)}$.

La formule de Leibniz implique que le membre de droite de l'égalité (4.16) s'écrit

$$
\frac{1}{\left(a_{1} \ldots a_{n}\right)^{n M}} \frac{1}{\mathcal{M} !} \mathcal{F}_{k}\left(\alpha_{1}, \ldots, \alpha_{n}, \frac{1}{\widetilde{R}(\alpha)}, \frac{1}{\theta_{1}\left(\alpha_{1}\right)}, \ldots, \frac{1}{\theta_{n}\left(\alpha_{n}\right)}\right)
$$


avec $\mathcal{F}_{k} \in \mathbb{Z}\left[z_{1}, \ldots, z_{2 n+1}\right] ;$ et si $d_{j}=\operatorname{deg}_{z_{j}} \mathcal{F}_{k}, 1 \leq j \leq 2 n+1$,

$$
\begin{aligned}
d_{j} & \leq \kappa(n) D^{2} \Delta^{2}\left(D_{Q}+\Delta\right)^{2}, \quad 1 \leq j \leq n, \\
d_{n+1} & \leq \kappa(n) D \Delta^{2}\left(D_{Q}+\Delta\right), \\
d_{n+1+j} & \leq \kappa(n) D \Delta^{2}\left(D_{Q}+\Delta\right), \quad 1 \leq j \leq n .
\end{aligned}
$$

D'après (4.9), un dénominateur d'un nombre algébrique de la forme (4.17) est

$$
\begin{aligned}
\left(a_{1} \ldots a_{n}\right)^{n M} \mathcal{M} ! & \left(\prod_{j=1}^{n}\left(\operatorname{den}\left(\alpha_{j}\right)\right)^{d_{j}}\right) \\
& \times\left(\operatorname{den}\left(\frac{1}{\widetilde{R}(\alpha)}\right)\right)^{d_{n+1}}\left(\prod_{j=1}^{n} \operatorname{den}\left(\frac{1}{\theta_{j}\left(\alpha_{j}\right)}\right)^{d_{n+1+j}}\right) .
\end{aligned}
$$

Il en découle qu'un dénominateur $\delta_{\alpha}$ commun aux nombres algébriques (4.13) est

$$
\begin{aligned}
& \left(a_{1} \ldots a_{n}\right)^{n M} \mathcal{M} ! \\
& \times\left\{\left(\prod_{j=1}^{n} \operatorname{den}\left(\alpha_{j}\right)\right)^{D\left(D_{Q}+\Delta\right)} \operatorname{den}\left(\frac{1}{\widetilde{R}(\alpha)}\right)\left(\prod_{j=1}^{n} \operatorname{den}\left(\frac{1}{\theta_{j}\left(\alpha_{j}\right)}\right)\right)\right\}^{\kappa(n) D \Delta^{2}\left(D_{Q}+\Delta\right)} .
\end{aligned}
$$

Maintenant, estimons les différents dénominateurs qui interviennent dans l'écriture de $\delta_{\alpha}$. Comme $B_{j}\left(\alpha_{j}\right)=0$, la valeur absolue du coefficient dominant de $B_{j}$ est un dénominateur de $\alpha_{j}$, donc

$$
\log \operatorname{den}\left(\alpha_{j}\right) \leq h\left(B_{j}\right) \leq \kappa(n) D^{2} \Delta^{2}(h+m \log D+D \log D) .
$$

Pour les autres dénominateurs nous avons besoin, d'après la propriété (4.8), d'estimer le degré et la taille des nombres algébriques $\widetilde{R}(\alpha), \theta_{1}\left(\alpha_{1}\right), \ldots$, $\theta_{n}\left(\alpha_{n}\right)$; leurs degrés sont au plus $\Delta$ ([BY1], Corollaire 2.2).

Puisque tous les conjugués de $\alpha_{j}$ sont solutions de $B_{j}\left(z_{j}\right)=0$ et toute solution $s$ d'une équation algébrique $a_{0} x^{d}+a_{1} x^{d-1}+\ldots+a_{d}=0$ vérifie $|s| \leq \max _{1 \leq i \leq d}\left|d a_{i} / a_{0}\right|^{1 / i}$, nous avons

(4.19) $\log \left|\bar{\alpha}_{j}\right| \leq h\left(B_{j}\right)+\log \operatorname{deg} B_{j} \leq \kappa(n) D^{2} \Delta^{2}(h+m \log D+D \log D)$.

Il résulte de (4.18) et (4.19) que

$$
\begin{aligned}
\mathrm{t}\left(\alpha_{j}\right) & =\max \left(\log \operatorname{den}\left(\alpha_{j}\right), \log \left|\bar{\alpha}_{j}\right|\right) \\
& \leq \kappa(n) D^{2} \Delta^{2}(h+m \log D+D \log D) .
\end{aligned}
$$

Par suite, d'après (4.10),

$$
\mathrm{t}(\widetilde{R}(\alpha)) \leq \kappa(n) D^{2} \Delta^{2}\left(D_{Q}+\Delta\right)(h+m \log D+D \log D),
$$


et d'après (4.8)

(4.21) $\log \operatorname{den}\left(\frac{1}{\widetilde{R}(\alpha)}\right) \leq \kappa(n) D^{2} \Delta^{3}\left(D_{Q}+\Delta\right)(h+m \log D+D \log D)$.

Pour la taille de $\theta_{j}\left(\alpha_{j}\right)$, nous remarquons que $\theta_{j}\left(\alpha_{j}\right)=B_{j}^{\left(\nu_{j}\right)}\left(\alpha_{j}\right) / \nu_{j}$ ! et la hauteur logarithmique du polynôme à coefficients entiers $B_{j}^{\left(\nu_{j}\right)}\left(z_{i}\right) / \nu_{j}$ ! est au plus $h\left(B_{j}\right)+\operatorname{deg} B_{j} \log 2$. En utilisant de nouveau (4.10) et (4.20),

$$
\mathrm{t}\left(\theta_{j}\left(\alpha_{j}\right)\right) \leq \kappa(n) D^{3} \Delta^{3}(h+m \log D+D \log D) .
$$

D'après la propriété (4.8),

$$
\log \operatorname{den}\left(\frac{1}{\theta_{j}\left(\alpha_{j}\right)}\right) \leq \kappa(n) D^{3} \Delta^{4}(h+m \log D+D \log D) .
$$

Nous déduisons, de (4.18), (4.21) et (4.22), l'estimation suivante pour $\delta_{\alpha}$ :

$$
\log \delta_{\alpha} \leq \kappa(n) D^{4} \Delta^{5}\left(D_{Q}+\Delta\right)^{2}(h+m \log D+D \log D) .
$$

Le nombre total de $\alpha$ intervenant dans (4.11) est au plus $\kappa(n) \Delta$, donc l'entier $\delta=\prod_{\alpha} \delta_{\alpha}$ est un dénominateur commun aux coefficients des polynômes $Q_{1}, \ldots, Q_{m} ;$ sa taille vérifie donc

$$
\log \delta \leq \kappa(n) D^{4} \Delta^{6}\left(D_{Q}+\Delta\right)^{2}(h+m \log D+D \log D) .
$$

Les quotients $Q_{1}, \ldots, Q_{m}$ qui apparaissent dans l'identité (4.7) sont en fait à coefficients rationnels. En effet, soient $\alpha_{1}, \ldots, \alpha_{s}$ les zéros communs aux polynômes $L_{1} p_{1}, \ldots, L_{n} p_{n}$, non zéros communs aux polynômes $P_{1}, \ldots, P_{m}$. Soit $A=\left\{\alpha_{11}, \ldots, \alpha_{1 n}, \ldots, \alpha_{s 1}, \ldots, \alpha_{s n}\right\}$, où $\alpha_{i 1}, \ldots, \alpha_{i n}$ sont les composantes de $\alpha_{i}, 1 \leq i \leq s$, et soit $\bar{A}$ l'ensemble des $\mathbb{Q}$-conjugués de tous les éléments de $A$. Etant donné un automorphisme $\sigma$ du groupe de Galois de l'extension $\mathbb{Q}(\bar{A}) / \mathbb{Q}$, alors

$$
\sigma\left(\left\langle\bar{\partial}\left(1 / L^{M} p\right), \frac{\zeta^{k}}{R(\zeta)} d \zeta\right\rangle_{\alpha}\right)=\left\langle\bar{\partial}\left(1 / L^{M} p\right), \frac{\zeta^{k}}{R(\zeta)} d \zeta\right\rangle_{\beta}
$$

où $\beta$ est, comme $\alpha$, un zéro commun à $L_{1} p_{1}, \ldots, L_{n} p_{n}$ non zéro commun à $P_{1}, \ldots, P_{m} ;$ car d'après la loi de transformation

$$
\left\langle\bar{\partial}\left(1 / L^{M} p\right), \frac{\zeta^{k}}{R(\zeta)} d \zeta\right\rangle_{\alpha}
$$

est une fraction rationnelle en $\alpha=\left(\alpha_{1}, \ldots, \alpha_{n}\right)$ à coefficients entiers. Faire agir l'automorphisme $\sigma$ revient à substituer dans cette fraction rationnelle $(\sigma(\alpha))_{j}$ à $\alpha_{j}$. Puisque $\alpha$ est un zéro de $L_{1} p_{1}, \ldots, L_{n} p_{n}$ et que $\sigma$ est un automorphisme, il en est de même de $\beta=\sigma(\alpha)$; pour les mêmes raisons, on a, puisque $P_{1} \ldots P_{m}(\alpha) \neq 0$, également $P_{1} \ldots P_{m}(\beta) \neq 0$. Les polynômes $Q_{1}, \ldots, Q_{m}$ intervenant dans $(4.7)$ sont à coefficients dans $\mathbb{Q}(\bar{A})$; ce qui précède montre qu'ils sont invariants sous l'action du groupe de Galois de 
l'extension $\mathbb{Q}(\bar{A}) / \mathbb{Q}$. Nous pouvons alors écrire la formule (4.7) sous la forme suivante :

$$
\delta Q^{n}=\sum_{k=1}^{m} P_{k} A_{k}, \quad A_{k} \in \mathbb{Z}\left[z_{1}, \ldots, z_{n}\right], 1 \leq k \leq m .
$$

Il nous reste à estimer la taille des coefficients des polynômes $A_{1}, \ldots, A_{m}$. Pour cela, cherchons une borne pour les nombres algébriques

$$
\begin{aligned}
\sum_{\substack{\alpha \in \mathbb{C}^{n}: p_{i}(\alpha)=0, i \in J \\
L_{j}(\alpha)=0, j \notin J \\
\Sigma_{l=1}^{m}\left|P_{l}(\alpha)\right|>0}}\left\langle\left(\bigwedge_{i \in J} \bar{\partial}\left(1 / \dot{p}_{i}\right) \bigwedge_{j \notin J} \bar{\partial}\left(1 / L_{j}^{M}\right)\right)^{\prime}, \frac{\zeta^{k}}{\widetilde{R}(\zeta)} d \zeta\right\rangle_{\alpha}, \\
|k| \leq \kappa(n)\left(D_{Q}+\Delta\right) .
\end{aligned}
$$

Lorsque $J \neq\{1, \ldots, n\}$, l'expression (4.24) n'est autre que le résidu global de $\zeta^{k} / \widetilde{R}(\zeta)$ relatif à l'application polynomiale $\Phi=\left(\dot{p}_{i}, i \in J ; L_{j}, j \notin J\right)^{\prime}$.

En appliquant l'inégalité diophantienne du type Łojasiewicz de Brownawell [B2] à $\widetilde{R}, \Phi_{1}, \ldots, \Phi_{n}$ et $\alpha$ appartenant à

$$
W=\left\{\zeta \in \mathbb{C}^{n}: \Phi_{1}(\zeta)=\ldots=\Phi_{n}(\zeta)=0, \sum_{i=1}^{m}\left|P_{i}(\zeta)\right|>0\right\}
$$

nous déduisons la borne inférieure suivante pour $|\widetilde{R}(\alpha)|$ :

$$
|\widetilde{R}(\alpha)| \geq \exp \left(-\kappa(n) D^{2} \Delta^{2}\left(D_{Q}+\Delta\right)^{2}(h+m \log D+D \log D)\right)=\eta .
$$

La même inégalité (avec une constante $\kappa(n)$ légèrement différente) reste valable dans le disque $B(\alpha, \eta)$ de centre $\alpha$ et de rayon $\eta$. Le cardinal de $W$ est au plus $\Delta$; donc si l'on divise $B(\alpha, \eta)$ en $\Delta+1$ couronnes concentriques de même épaisseur, une au moins de ces couronnes ne contient pas d'éléments de $W$. Notons $S_{\alpha}$ la sphère médiane de celle-ci; nous avons

$$
\forall \zeta \in S_{\alpha}, \quad d(\zeta, W) \geq \frac{\eta}{2(\Delta+1)} .
$$

Nous fixons $\zeta \in S_{\alpha}$ et nous appliquons à nouveau les inégalités diophantiennes du type Łojasiewicz de Brownawell; il vient

$$
\log \max _{1 \leq i \leq n}\left|\Phi_{i}(\zeta)\right| \geq-\kappa(n) D^{2} \Delta^{3}\left(D_{Q}+\Delta\right)^{2}(h+m \log D+D \log D) .
$$

Soit $g=\left(\dot{p}_{i}, i \in J ; L_{j}^{M}, j \notin J\right)$, nous avons donc pour tout $\alpha \in W$ et pour tout $\zeta \in S_{\alpha}$,

$$
\log \|g(\zeta)\| \geq-\kappa(n) D^{2} \Delta^{3}\left(D_{Q}+\Delta\right)^{3}(h+m \log D+D \log D) .
$$

Désignons par $B_{\alpha}$ la boule fermée dont la frontière est $S_{\alpha}$. Nous ordonnons la famille de ces boules de façon que les rayons soient décroissants. Considérons 
les domaines

$$
\Omega_{1}=B_{1}, \quad \Omega_{2}=B_{2} \backslash B_{1}, \quad \Omega_{3}=B_{3} \backslash\left(B_{1} \cup B_{2}\right), \quad \ldots
$$

qui contiennent des éléments de $W$. D'après leur construction, ils sont disjoints, $W \subset \bigcup_{l} \stackrel{\circ}{\Omega}_{l}$, et l'aire de la surface $\partial \Omega_{l}$ est bornée par $\omega_{2 n-1} \eta^{2 n-1} \Delta$, $\omega_{2 n-1}$ étant l'aire de la sphère unité de $\mathbb{C}^{n}$. Il s'ensuit que les expressions (4.24) sont égales à

$$
\sum_{l}\left(\sum_{\alpha \in V \cap \Omega_{l}}\left\langle\left(\bigwedge_{i \in J} \bar{\partial}\left(1 / \dot{p}_{i}\right) \bigwedge_{j \notin J} \bar{\partial}\left(1 / L_{j}^{M}\right)\right)^{\prime}, \frac{\zeta^{k}}{\widetilde{R}(\zeta)} d \zeta\right\rangle_{\alpha}\right) .
$$

Les quantités entre parenthèses de l'expression (4.25) se calculent par l'intermédiaire des formules de Bochner-Martinelli ([GH], $[\mathrm{T}])$; elles sont égales à

$$
\frac{(n-1) !}{(2 \pi i)^{n}} \int_{\partial \Omega_{l}} \frac{\zeta^{k}}{\widetilde{R}(\zeta)} \frac{1}{\|g(\zeta)\|^{2 n}}\left(\sum_{j=1}^{n}(-1)^{j-1} \overline{g_{j}} \bigwedge_{\substack{l=1 \\ l \neq j}}^{n} \overline{\partial g_{l}} \wedge d \zeta\right) .
$$

En regardant les différents termes intervenant dans (4.26), nous déduisons la borne suivante pour les nombres algébriques (4.11) :

$$
\exp \left(\kappa(n) D^{2} \Delta^{3}\left(D_{Q}+\Delta\right)^{3}(h+m \log D+D \log D)\right) .
$$

Finalement, puisque nous savons estimer tous les membres qui apparaissent dans l'expression des polynômes $Q_{1}, \ldots, Q_{m}$, nous déduisons l'estimation suivante pour la taille des coefficients des quotients $A_{1}, \ldots, A_{m}$ :

$$
\max _{1 \leq i \leq m} h\left(A_{i}\right) \leq \kappa(n)\left(D^{4} \Delta^{5}\left(D_{Q}+\Delta\right)^{3}(h+m \log D+D \log D)+h_{Q}\right),
$$

ce qui achève la preuve du théorème 4.5 .

\section{Références}

[BGVY] C. A. Berenstein, R. Gay, A. Vidras and A. Yger, Residue Currents and Bézout Identities, Progr. Math. 114, Birkhäuser, 1993.

[BY1] C. A. Berenstein and A. Yger, Effective Bézout identities in $\mathbb{Q}\left[z_{1}, \ldots, z_{n}\right]$, Acta Math. 166 (1991), 69-120.

[BY2] -, -, Formules de représentation intégrale et problèmes de division, dans : Approximations Diophantiennes et Nombres Transcendants, Luminy 90, P. Philippon (éd.), Walter de Gruyter, Berlin, 1992, 15-37.

[BGS1] J.-B. Bost, H. Gillet et C. Soulé, Un analogue arithmétique du théorème de Bézout, C. R. Acad. Sci. Paris 312 (1991), 845-848.

[BGS2] - - - - , Heights of projective varieties and positive Green-forms, J. Amer. Math. Soc. (1994) (à paraître).

[BS] J. Briançon et H. Skoda, Sur la clôture intégrale d'un idéal de germes de fonctions holomorphes en un point de $\mathbb{C}^{n}$, C. R. Acad. Sci. Paris 278 (1974), 949-951.

[B1] W. D. Brownawell, Bounds for the degrees in the Nullstellensatz, Ann. of Math. 126 (1987), 577-591. 
[B2] W. D. Brownawell, Local diophantine Nullstellen inequalities, J. Amer. Math. Soc. 1 (1988), 311-322.

[CGH] L. Caniglia, A. Galligo et J. Heintz, Borne simple exponentielle pour les degrés dans les théorèmes des zéros sur un corps de caractéristique quelconque, C. R. Acad. Sci. Paris 307 (1988), 255-258.

[E] M. Elkadi, Bornes pour les degrés et les hauteurs dans le problème de division, Michigan Math. J. 40 (1993), 609-618.

[GS] H. Gillet and C. Soulé, Arithmetic intersection theory, Publ. Math. I.H.E.S. 72 (1990), 93-174.

[GH] P. Griffiths and J. Harris, Principles of Algebraic Geometry, Wiley-Interscience, New York, 1978.

[Hé] A. Hénaut, Cycle exceptionnel de l'éclatement d'un idéal définissant l'origine de $\mathbb{C}^{n}$ et applications, Ann. Inst. Fourier (Grenoble) 37 (3) (1987), 143-157.

[H] L. Hörmander, An Introduction to Complex Analysis in Several Variables, NorthHolland, Amsterdam, 1973.

[JKS] S. Ji, J. Kollár and B. Shiffman, A global Eojasiewicz inequality for algebraic varieties, Trans. Amer. Math. Soc. 329 (1992), 813-818.

[K] J. Kollár, Sharp effective Nullstellensatz, J. Amer. Math. Soc. 1 (1988), 963-975.

[LT] M. Lejeune et B. Teissier, Quelques calculs utils pour la résolution des singularités, Séminaire Ecole Polytechnique (1972), page 130.

[NR] D. G. Northcott and D. Rees, Reductions of ideals in local rings, Proc. Cambridge Philos. Soc. 50 (1954), 145-158.

[P] P. Philippon, Dénominateurs dans le théorème des zéros de Hilbert, Acta Arith. 58 (1991), 1-25.

[T] A. K. Tsikh, Multidimensional Residues and Their Applications, Transl. Math. Monographs 103, Amer. Math. Soc., 1992.

[V] B. L. van der Waerden, Algebra, Springer, New York, 1959.

[W] M. Waldschmidt, Un premier cours sur les nombres transcendants, Université Paris XI, N ${ }^{\circ} 47$, UER Mathématiques, 1973.

UNIVERSITÉ BORDEAUX I (CEREMAB)

351 COURS DE LA LIBÉRATION

33405 TALENCE

FRANCE 